\title{
Quantum mechanical understanding of field dependence of the apex barrier of a single-wall carbon nanotube
}

\author{
Jie Peng, Zhibing Li, Chunshan He, Shaozhi Deng, and Ningsheng $\mathrm{Xu}^{*}$ \\ The State Key Laboratory of Optoelectronic Materials and Technologies, Department of Physics, Zhongshan University, \\ Guangzhou, 510275, People's Republic of China \\ Xiao Zheng and GuanHua Chen ${ }^{\dagger}$ \\ Department of Chemistry, The University of Hong Kong, Hong Kong, China \\ (Received 23 May 2005; revised manuscript received 8 August 2005; published 2 December 2005)
}

\begin{abstract}
The potential barrier at the apex of a single-wall carbon nanotube emitter is found to be strongly and nonlinearly dependent on the external applied field, due to a quantum mechanical mechanism instead of the correction of image potential in Fowler-Nordheim theory. The field enhancement factor depends on the applied field and is much smaller than that predicted by the classical theory. The field induced apex-vacuum barrier lowering is confirmed to be the essential mechanism for efficient field electron emission from capped carbon nanotubes.
\end{abstract}

DOI: 10.1103/PhysRevB.72.235106

PACS number(s): 71.15.-m, 73.22.-f

\section{INTRODUCTION}

Excellent field emission features of carbon nanotubes (CNTs) have been observed in a number of experiments. ${ }^{1-3}$ It is not surprising since it is expected that the high aspect ratio of CNTs would lead to a great enhancement of the local electric field near the tip, which would reduce significantly the barrier between the CNTs and the vacuum and make the field emission easier. In order to explain the experimental results by the Fowler-Nordheim (FN) theory, ${ }^{4}$ one needs a field enhancement factor as large as several thousands for a CNT whose work function is about $5.0 \mathrm{eV}$. Although this is consistent with the electrostatics conclusion that the field enhancement factor for a long metal rod is approximately the same as its aspect ratio, some problems remain unclarified. (1) Can the CNTs shield the electrostatic field completely as a metal rod? If not, the field enhancement would depend on the structural properties of the CNT in a complicated way. (2) According to the classical theory, the CNTs under applied fields would become unstable as the tube length exceeds a certain value when the field strength at the tip is comparable to the atomic field due to the huge field enhancement. As a stable field emission from CNTs of several micrometers has been observed in experiments, the classical theory must fail for long CNTs. (3) Is there any unknown quantum effect that plays a role in the nanoscale tube tip? Since it was found that field emission is closely related to the local electronic states in the tip. ${ }^{5,6}$ (4) In the classical FN theory, the potential barrier between a metal and the vacuum is a combination of the ramp potential of the applied field and the image potential of the emission electron. The latter arises from a macroscopic mean effect and is found to be field independent. As the apex of the CNT is in nanoscale, the concept of image potential is no longer applicable. To clarify these issues, we need to know the detailed electronic structure of the CNTs under applied external fields, especially the charge distribution and the potential barrier in the tip region

A quantum mechanical investigation is thus necessary. However, as a carbon nanotube is a huge molecule which consists of hundreds of thousands to millions of carbon atoms, an atomic level calculation of the entire tube is still far from achievable. Most of the previous calculations based on density-functional theory ${ }^{7-9}$ (DFT) were carried out on nanotubes whose lengths are only a few nanometers because of the limitation of computational resources. To compare with experimental results, a factor $L_{\text {tube }} / L_{\text {tip }}$ is introduced to scale the external field in the simulation on a short $\mathrm{CNT}^{7}{ }^{7}$ where $L_{\text {tube }}$ represents the length of the tube of experimental usage while $L_{\text {tip }}$ is the length adopted in the calculation. Based on this simplified model, some suggestive results have been obtained. However, the potential barrier is sensitive to the charge accumulation along the tube body because of the long-range nature of the Coulomb interaction. In addition, the possible field penetration at the tube tip would lower significantly the field enhancement factor. ${ }^{10}$ Therefore, a fullsized simulation is appealing.

A multiscale strategy is a solution to this complex problem. The idea is to divide the total system into two regions and then treat different regions with methods of different scales. The region near the tip is simulated quantum mechanically $(\mathrm{QM})$, while the region far from the tip is simulated by molecular mechanics (MM),${ }^{11}$ as the field emission characteristic is not sensitive to the electronic structure in the region far from the tip. The potential barrier for emission is obtained directly by the calculated charge distribution along the entire tube. Recently, simulations on a $1 \mu \mathrm{m}$ long fieldemitting single-walled carbon nanotube (SWCNT) has been successfully carried out by the combined quantum mechanics and molecular mechanics (QM/MM) approach. ${ }^{10} \mathrm{~A}$ new mechanism has been proposed in Ref. 10 to account for the exceptional emission characteristics of CNTs, which is the lowering of the potential barrier with the increase of the applied field.

The present article is an extension of Ref. 10. In order to predict more characteristics of the field electron emission from CNTs, which would be compared with experimental results, we make a more comprehensive investigation on 


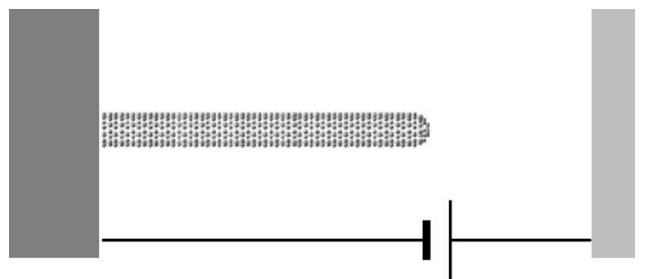

FIG. 1. Setup for field emission from a SWCNT. The SWCNT is vertically mounted on the metallic cathode. The distance between the cathode and anode is unphysical.

capped $(5,5)$ SWCNTs. In the present paper we have removed the effect of the artificial charges induced at each subsystem due to the saturation of the dangling bonds, which makes sense when artificial charges are comparable to the induces charges in the subsystem. The emission current versus the tube length is obtained. The field enhancement factor is extracted directly from the simulated local electric field distribution. We confirm the new mechanism for CNT field emission proposed by Ref. 10. Furthermore, the potential barrier as a step function of the applied field is revealed. This observation should be related to the local density of states in the tip region and have a quantum origin. The potential barrier is the superposition of potentials of the core ions, valence electrons, and induced electrons in the tip region.

In Sec. II, we describe in detail the physical mechanism for the field emission from micrometer-long CNTs. The simulation methodology is introduced in Sec. III. Simulation results and the related interpretations are presented in Sec. IV, and conclusions are drawn in Sec. V.

\section{THE MECHANISM}

Figure 1 depicts a typical field emission setup. An SWCNT is vertically mounted on a flat metallic cathode. Specifically we consider a SWCNT of $(5,5)$ chirality, ${ }^{12}$ which is a metallic tube presumably. Since the SWCNT of our interest is as long as micrometers, the possible Schottky junction at the interface of the cathode and the SWCNT can be neglected. In the equilibrium, the Fermi level (or the chemical potential) is a constant over the entire SWCNT and is equal to that of the cathode. Throughout this paper, we will assume that the SWCNT is in equilibrium, i.e., the so-called quasiequilibrium approximation is adopted. This is reasonable before the SWCNT emits or when the emission current is feeble. John Cumings et al. ${ }^{13}$ have observed that the electric field distribution varies less than $2.5 \%$ while the emission current undergoes a fluctuation of $80 \%$. This implies that the current has little effect on the potential and electron density distribution at the tube tip. Hence the image effect of emitting electrons is also neglected.

Driven by an applied field, the redistribution of the electrons leads to two major consequences. (1) The electric field is shielded inside the tube body, which results in an enhancement of the local electric field at the tube tip. However, since a SWCNT consists of only one layer of carbon atoms, the shielding is by no means complete. There is field penetration in the tip region. The field enhancement is thus less efficient

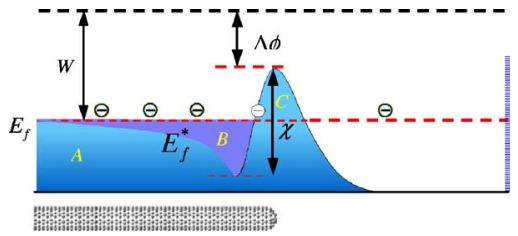

FIG. 2. (Color online) A zone: filled with atomic electrons; B zone: filled with induced electrons; $\mathrm{C}$ zone: the barrier between the CNT and the vacuum. $\mu$ is the chemical potential of the joint system, $E_{f}^{*}$ is the intrinsic Fermi level at the tip, $W$ is the work function of the cathode, $\chi$ is the electron affinity of the CNT, and $\Delta \phi$ is the barrier lowering.

than that for a classical metal rod. (2) There are excess charges accumulating along the tube, especially in the tip region. In the quasiequilibrium approximation, the intrinsic Fermi level of the SWCNT (i.e., the Fermi level of the isolated neutral SWCNT) bends down due to the lowering of local orbital energies by the applied field. Excess charges can thus be accommodated in the orbitals with the energy between the chemical potential of the joint system (the SWCNT plus the cathode) and the intrinsic Fermi level of the SWCNT. A schematic picture is given in Fig. 2. When electrons fill the A zone, the SWCNT is neutral. Each electron in the orbital in the B zone contributes a charge of $-e$, which forms the induced charge distribution. The curve separating the $\mathrm{A}$ zone and the $\mathrm{B}$ zone is the intrinsic Fermi level $E_{f}^{*}$, The barrier between the CNT and the vacuum is labeled by C. $W$ is the work function of the cathode, and $\chi$ is the electron affinity of the SWCNT.

When an external field is applied between the cathode and the anode, the bending of the intrinsic Fermi level of the SWCNT leads to the lowering of the vacuum barrier, which is denoted by $\Delta \phi$ in Fig. 2 . The electrons at the tube tip are thus confined by a potential much lower than the electron affinity of an isolated SWCNT. The barrier lowering corresponding to the charge accumulation at the tip is a new mechanism that favors the field emission in addition to the well-known field enhancement effect. ${ }^{11}$ For a neutral armchair SWCNT, it is believed that the molecular orbitals below the intrinsic Fermi level are $\pi$ orbitals and those above are of $\pi^{*}$ symmetry. ${ }^{12}$ In the applied field, as mentioned above, some $\pi^{*}$ orbitals sink below the chemical potential of the joint system which are subsequently occupied by excess charges from the cathode substrate. Therefore the emitted electrons come most probably from the $\pi^{*}$ orbitals near the chemical potential of the joint system.

\section{THE SIMULATION METHOD}

The SWCNT in our model is taken as a quantum object subject to the following boundary condition:

$$
V=\left\{\begin{array}{c}
V_{a}>0, \text { anode }, \\
0, \text { cathode } .
\end{array}\right.
$$

The cathode is a flat metallic plate. The voltage drop from the anode to the cathode is fixed as $V_{a}$.

To simulate the entire tube, we adopt the hybrid QM/MM approach described in Ref. 10. The entire CNT is first di- 


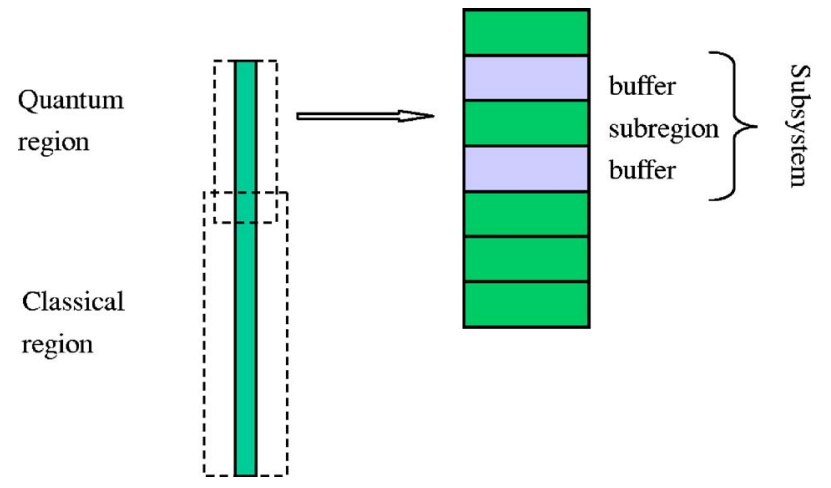

FIG. 3. (Color online) The quantum region and classical region of a CNT. The quantum region is divided into subregions. Each subregion and its neighbors form a subsystem.

vided into two parts, a quantum part and a classical part (see Fig. 3). In the quantum part all valence electrons and excess electrons induced by the applied field are treated quantum mechanically. The classical part is the rest of the CNT in which the electrons and core ions are taken as point charges since their predominant influence on the emission is through their electrostatic potential at the tip.

We employ the linear-scaling divide-and-conquer (DAC) method ${ }^{16}$ to calculate the charge distribution in the quantum region. The DAC method divides the quantum region into some subregions (40 atoms in each subregion in our simulation). We want the separation error of each subsystem to have similar magnitude, so the size of the top subregion is doubled since it has only one buffer. The subregion and its adjacent subregions (referred to as buffers) form a subsystem $\Omega$ that is to be simulated by an $a b$ initio method, as magnified schematically on the right-hand side (RHS) of Fig. 3. The two buffers (only one buffer for the top subregion) are used to reduce the error of separating the subregion from the CNT. The dangling bonds at the end of buffers are saturated by hydrogen atoms to ensure the convergence of selfconsistent field (SCF). The Hamiltonian of the subsystem $\Omega$ can be explicitly written as

$$
\begin{aligned}
\hat{H}^{\Omega}= & -\frac{1}{2} \sum_{i \in \Omega}^{N_{\Omega}} \nabla_{i}^{2}-\sum_{i \in \Omega}^{N_{\Omega}} \sum_{A \in \Omega}^{M_{\Omega}} \frac{Z_{A}}{r_{i A}}+\sum_{\substack{i<j \\
i, j \in \Omega}}^{N_{\Omega}} \frac{1}{r_{i j}} \\
& -\sum_{i \in \Omega}^{N_{\Omega}} \sum_{B \in \bar{\Omega}} \frac{Z_{B}}{r_{i B}}-\sum_{\substack{i \in \Omega \\
C \in \text { image }}} \frac{Z_{C}}{r_{i C}}-E_{0} \sum_{i \in \Omega}^{N_{\Omega}} z_{i},
\end{aligned}
$$

where $N_{\Omega}$ and $M_{\Omega}$ represent, respectively, the number of electrons and the number of core ions in the subsystem $\Omega$. The first term on the RHS is the kinetic energy of electrons in $\Omega$, the second is the Coulomb interaction between electrons and the core ions in $\Omega$, the third is the interaction between electrons in $\Omega$, the fourth term is the interaction between the electrons in $\Omega$ and the extra charges not in $\Omega$, the fifth term accounts for the electrostatic potential due to the presence of the image charges, and the last term on the RHS arises directly from the applied field. With Eq. (2), the molecular orbitals of the $\Omega$ subsystem are calculated for a given $N_{\Omega}$.

In the linear combination of atomic orbitals (LCAO) approximation, the reduced single-electron density matrix of the subregion $\alpha$ is

$$
\rho_{\gamma \delta}^{\alpha}=P_{\gamma \delta}^{\alpha} \sum_{i} f_{\beta}\left(\mu-\epsilon_{i}^{\alpha}\right)\left(c_{\gamma i}^{\alpha}\right)^{*} c_{\delta i}^{\alpha}
$$

where $f_{\beta}$ is the function of Fermi distribution, $\mu$ is the chemical potential of the joint system, while $c_{\gamma i}^{\alpha}$ and $\epsilon_{i}^{\alpha}$ are local eigenvectors and eigenvalues of the subsystem. The partition matrix $P_{\gamma \delta}^{\alpha}$ satisfies the normalization condition and can be constructed in a simple way, ${ }^{16}$

$$
P_{\gamma \delta}^{\alpha}=\left\{\begin{array}{c}
1 \text { if } \gamma \in \alpha, \delta \in \alpha, \\
1 / 2 \text { if } \gamma \in \alpha, \delta \notin \alpha, \\
0 \text { if } \gamma \notin \alpha, \delta \notin \alpha .
\end{array}\right.
$$

The information of the other subregion that is not adjacent to the subregion under consideration can partly be included in the local density matrix through the fourth and fifth terms of the RHS of Eq. (2). The charge distribution of the MM part also affects the subregions in QM part through Coulomb interaction. Based on the fact that density of states (DOS) near the intrinsic Fermi energy of a SWCNT is approximately constant, ${ }^{14,15}$ Ref. 10 has deduced the linear induced charge density for a small shift of intrinsic Fermi energy

$$
\rho(z)=\frac{\rho(z)}{\sinh \left(\frac{L^{\prime}}{\lambda}\right)} \sinh \left(\frac{z}{\lambda}\right),
$$

where $\rho\left(L^{\prime}\right)$ is the linear induced charge density at $z=L^{\prime}$ and $\lambda$ is the characteristic decay length of the induced charge. Both $\rho\left(L^{\prime}\right)$ and $\lambda$ should be determined by the quantum mechanical simulation.

The major cycle is about the entire QM part. The subsystems are simulated one by one from the tube tip and the results provide the parameters $\rho\left(L^{\prime}\right)$ and $\lambda$, which are transferred to the MM part through Eq. (5). A lesser cycle for the decision of $N_{\Omega}$ of a subsystem is in a given applied field and charge environment. $N_{\Omega}$ varies until the Fermi energy converges to the preset chemical potential. Usually several rounds are needed to reach this equilibrium criterion. In each major cycle, the parameters $Z_{B}, Z_{C}$, and $N_{\Omega}$ of Eq. (2), as well as $\rho\left(L^{\prime}\right)$ and $\lambda$ of Eq. (5), are updated. The major cycle is repeated until a satisfactory convergency of all parameters is reached.

The simulation time is mostly spent on the quantum region. The quantum region is chosen so that the final results are not sensitive to its size and the fluctuation in the parameters $\lambda$ and $\rho\left(L^{\prime}\right)$ becomes negligibly small. According to our simulation, the induced charge distribution changes little with the atom number in the QM part as it varies from 4000 to 10000 in the applied field of $17 \mathrm{~V} / \mu \mathrm{m}$, while the distribution fluctuates drastically as the number varying in the range of 1000 to 2000 . Therefore in our real simulation, the QM part consists of 6000 atoms. In fact, for lower applied fields, the QM part could have fewer atoms. 

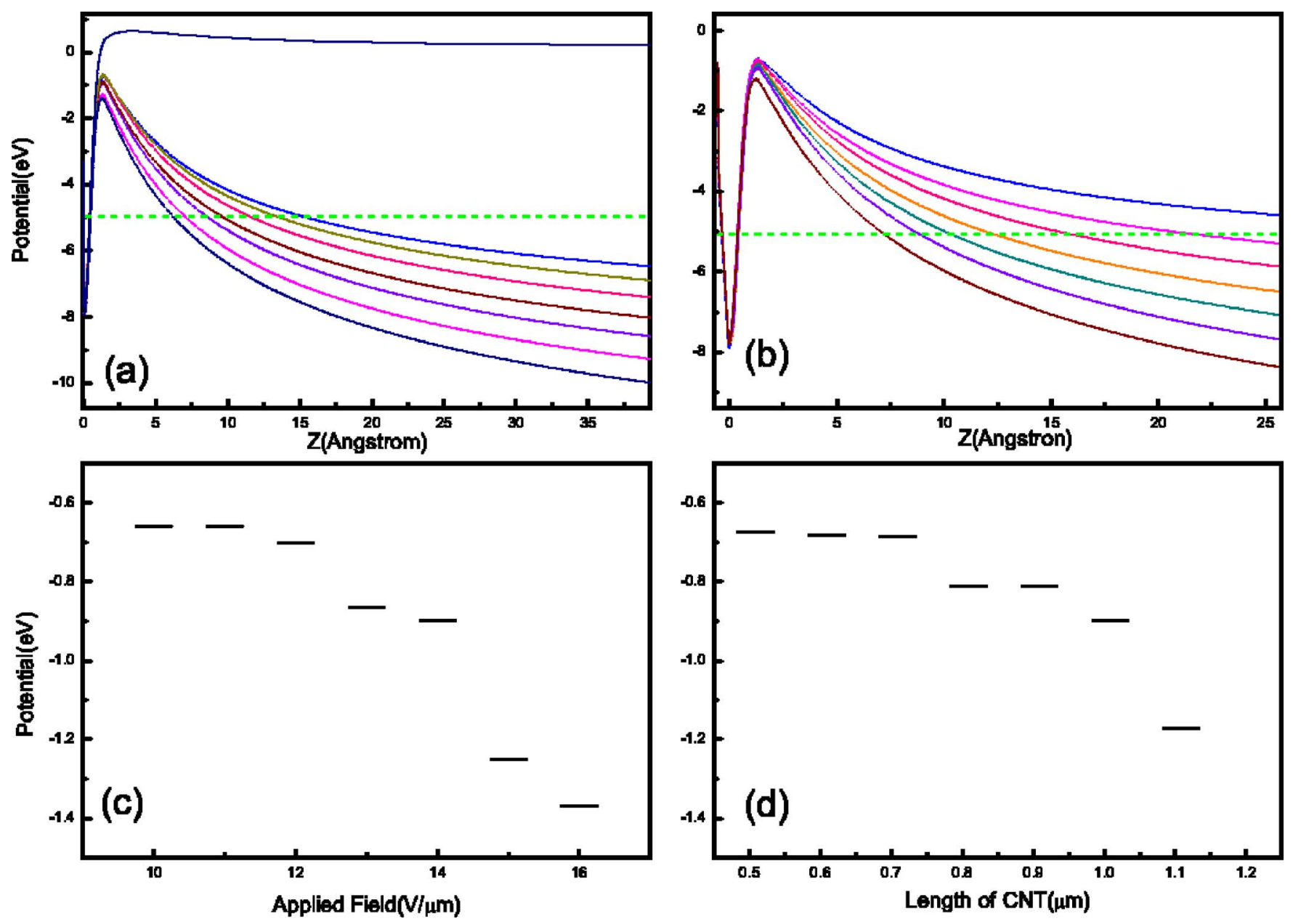

FIG. 4. (Color online) (a) The potentials in front of the capped (5,5) SWCNT in applied fields from 10.0 to $16.0 \mathrm{~V} / \mu \mathrm{m}$. The length of the SWCNT is fixed as $1.0 \mu \mathrm{m}$. (b) The potentials of SWCNTs of lengths from 0.5 to $1.1 \mu \mathrm{m}$. The applied field is fixed as $14.0 \mathrm{~V} / \mu \mathrm{m}$. The $z$ axis is the central axis of the SWCNTs with zero at the apex of the tubes. The dotted line is the Fermi level. (c) The barrier height versus applied fields. The length of CNT is $1.0 \mu \mathrm{m}$. (d) The barrier height versus CNT lengths. The applied field is $14.0 \mathrm{~V} / \mu \mathrm{m}$.

The subsystem that consists of the subregion and two buffers (only one buffer for the apex subregion) is calculated by the modified neglect of diatomic overlap (MNDO) semiempirical quantum mechanical method. On the boundary of the subsystem, the carbon-carbon bonds ( $\mathrm{C}-\mathrm{C}$ bonds) are cut and saturated by hydrogen atoms. Due to the difference in the electron negativity between the carbon and hydrogen atoms, some electrons enter the inner subregion from the buffers. This artifact is eliminated by manually subtracting these polarized charges from the calculated electron density distribution on a neutral nanotube in the absence of external fields.

\section{SIMULATION RESULTS}

We simulate the armchair $(5,5)$ capped CNTs of various lengths ranging from 0.5 to $1.1 \mu \mathrm{m}$. The applied field is ranged from 10.0 to $16.0 \mathrm{~V} / \mu \mathrm{m}$. For the subtraction of the artificial charges, the SWCNTs free of applied field are also simulated. Each subregion consists of 40 atoms except for the apex subregion, which consists of 80 atoms.

\section{A. Barrier lowering}

The apex-vacuum barrier height and the field enhancement factor are two important quantities for the field emis- sion. For a capped $(5,5)$ SWCNT of length $1.0 \mu \mathrm{m}$, the variation of the apex-vacuum barrier versus the applied fields is shown in Fig. 4(a). The horizontal axis is along the tube axis, and the vertical axis is the electrostatic potential. The highest curve corresponds to the electrostatic potential profile in the absence of applied fields, with the vacuum potential infinitely away being asymptotic zero. The others from up to down are for applied fields from 10.0 to $16.0 \mathrm{~V} / \mu \mathrm{m}$. The dashed horizontal line indicates the Fermi level (the SWCNT and the cathode). The barriers for SWCNTs of various lengths ranging from 0.5 to $1.1 \mu \mathrm{m}$ (from up to down) are depicted in Fig. 4(b) for a fixed applied field of $14.0 \mathrm{~V} / \mu \mathrm{m}$. It is found that the barrier is not only field dependent, but also length dependent. As the applied field or the length increases, the thickness and also the height of the barrier decrease significantly. The barrier height versus the applied fields is given in Fig. 4(c) for the $1.0 \mu \mathrm{m}$ SWCNT, and Fig. 4(d) plots the barrier heights for various lengths with the applied field fixed at $14.0 \mathrm{~V} / \mu \mathrm{m}$. It is found that the barrier height does not decrease smoothly, but similar to step functions, which should be related to the steplike DOS of the SWCNT. 

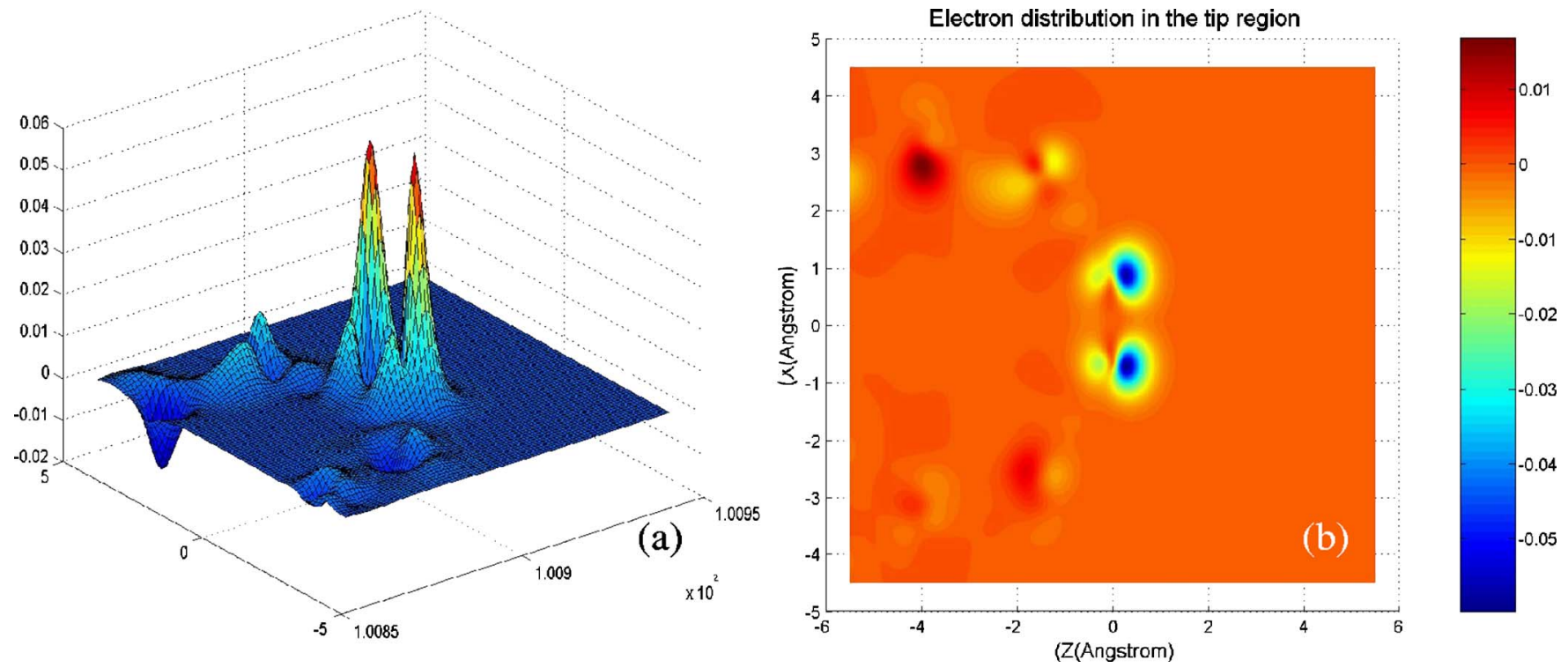

FIG. 5. (Color online) (a) The excess electron distribution in the section plane through the axis of the capped $(5,5)$ carbon nanotube under the applied field of $10.0 \mathrm{~V} / \mu \mathrm{m}$. (b) The two-dimensional map of charge distribution.

The lowering of the barrier caused by field penetration should be distinguished from the image effect of the field emission from a metal surface. The second differential of the image potential with respect to $z$ is negative while for SWCNT the correction to the linear potential has a positive second differential. It indicates that negative charge is induced in the SWCNT tip while the positive charge is responsible for the usual image effect.

Note that even without an applied field, there is still a smooth peak in the potential barrier in front of the apex of the tube, which is higher than the vacuum potential (zero). This is due to the boundary effect. As the uniform SWCNT is terminated by a half of a buckyball, the $\pi^{*}$ electrons have more probability to distribute outside the SWCNT due to the curvature of the cap. A dipole is thus created at the apex pointing to the cathode along the tube axis. This apex dipole is responsible for the small barrier peak in the absence of the applied field, and the applied field in turn strengthens the apex dipole. The electron distribution in a vertical section through the tube axis for the applied field of $10.0 \mathrm{~V} / \mu \mathrm{m}$ is presented in Fig. 5. We can see that the asymmetry of electron distribution near the first layer of carbon atoms that forms an electric dipole. Since the dipole is in the same direction as that of the applied field, the applied field outside the CNT wall is strengthened. This makes the potential barrier sharper, and hence it is a positive effect on the field electron emission. On the other hand, the net negative charges in front of the apex raise the barrier height because of the Coulomb repulsion, which is a negative effect of the field emission. However, the dipole effect is not comparable to the effect of barrier lowering due to the field penetration in the tip region.

\section{B. Emission current}

Reference 10 has shown that the potential barrier in the circumjacent of CNT is high and thick, so most probably the electrons emit forward from the first layer of carbon atoms at the tube tip. Assuming that most of the electrons emit from the Fermi level of the joint system $\mu$, we estimate the emission current by the WKB approximation

$$
I=\nu q_{\mathrm{exc}} D(\mu),
$$

where $q_{\mathrm{exc}}$ is the number of excess electrons in the first layer of atoms, and $\nu$ is the collision frequency that can be estimated from the average kinetic energy of $\pi^{*}$ electrons as $\nu$ $=E_{k}\left(\pi^{*}\right) / h$. Another way to estimate the collision frequency is to use the uncertainty relation

$$
\nu=\frac{E_{k}}{h}=\frac{h}{32 \pi^{2} m\left\langle\Delta r^{2}\right\rangle},
$$

where $\left\langle\Delta r^{2}\right\rangle$ is the uncertainty of the radial coordinate. Its numerical value can be estimated from the density of excess electrons (see Fig. 5). The collision frequencies estimated by two methods are of the same order of magnitude, i.e., $10^{14} \mathrm{~Hz}$. The transmission coefficient in the WKB approximation is

$$
D(\mu)=\exp \left[-\frac{2}{\hbar} \operatorname{Im} \int \sqrt{2 m[\mu-V(z)]} d z\right] .
$$

The computed current for the $1.0 \mu \mathrm{m}$ capped $(5,5)$ SWCNT is presented in Fig. 6. The inset shows the corresponding FN plot [4], which resembles a straight line. The dependence of the emission current on the CNT length in the range of $0.5-1.1 \mathrm{~V} / \mu \mathrm{m}$ is presented in Fig. 7, where the applied field is fixed at $14.0 \mathrm{~V} / \mu \mathrm{m}$. The crosses use the left axis in logarithmic scale, and the circles use the right axis.

\section{Field enhancement factor}

Although the FN plot of an individual SWCNT is roughly a straight line, the field emission mechanism is different from 


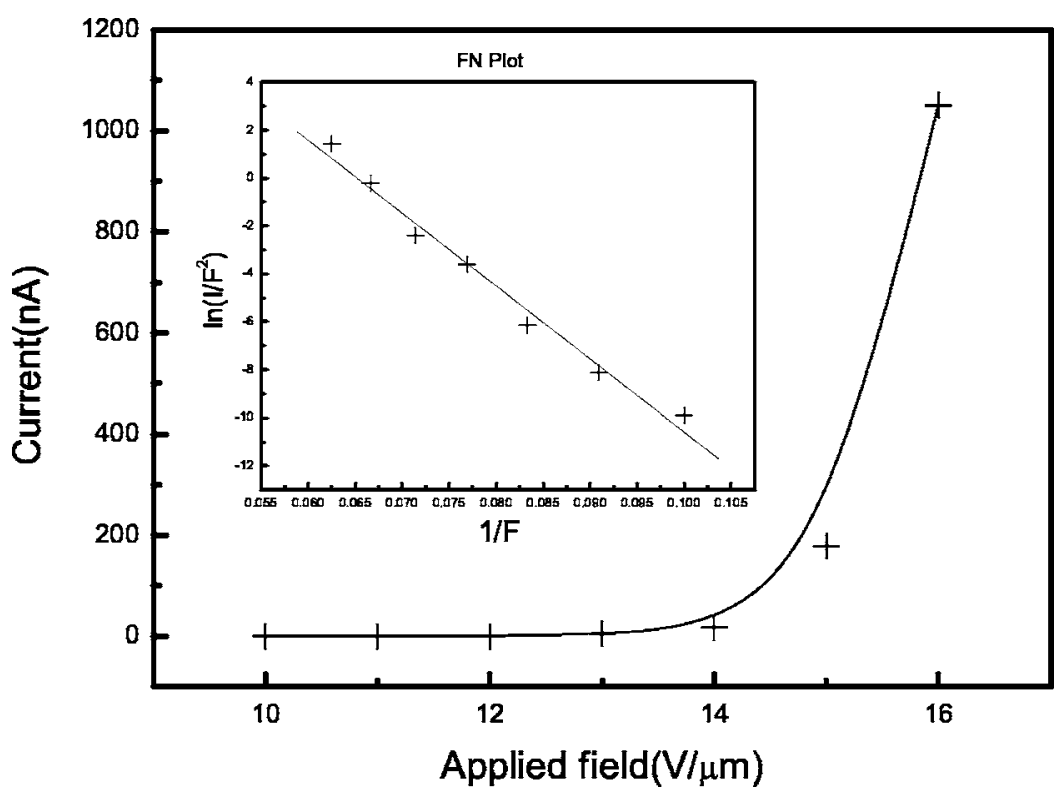

FIG. 6. The current versus applied fields characteristic curve of capped end $(5,5) \mathrm{CNT}$. The inset is the FN plot.

the classical FN theory as explained in Sec. II. The discrepancy in the field enhancement factor is apparent. In the classical FN theory, the field enhancement factor depends only on the geometrical structure of the emitter. However, in our simulation it is found that the field enhancement factor depends also on the applied field.

Here we should be careful of the different definitions of the field enhancement factor. One usually extracts the field enhancement factor from the experimental slope of the FN plot based on the FN theory. It will be referred to as the phenomenological field enhancement factor and denoted by $\gamma_{\text {phe }}$. Assuming that the classical FN theory is still applicable, i.e., the emitter could be considered as a metal plate, so

$$
\gamma_{\text {phe }}=-\frac{6.44 \times 10^{9} W^{3 / 2}}{K},
$$

where $K$ is the slope of the $\mathrm{FN}$ plot and $W$ is the work function $[5.0 \mathrm{eV}$ for the $(5,5) \mathrm{SWCNT}]$. The phenomeno- logical enhancement factor $\gamma_{\text {phe }}$ obtained by fitting our simulation results is 260, which is consistent with the existing experiments of an individual CNT [2]. But one should bear in mind that $\gamma_{\text {phe }}$ is neither the actual field enhancement factor nor the classical field enhancement factor but an effective one. The field enhancement factor by definition is the ratio of the electrostatic field and the applied field at the emission point. So far, this factor is only estimated by classical methods and will be referred to as $\gamma_{\mathrm{cl}}$. A classical calculation ${ }^{17}$ gives

$$
\gamma_{\mathrm{cl}}=5.93+0.73(L / R)-0.0001(L / R)^{2},
$$

which is numerically consistent with the earlier result of Ref. 18. For the $1.0 \mu \mathrm{m}(5,5)$ SWCNT, it is 1206.0, much larger than $\gamma_{\text {phe }}$. This indicates the failure of the classical theory. Since the full-size simulation for a SWCNT is carried out in the present article, the field enhancement factor can be extracted directly from the local electric field in the vicinity

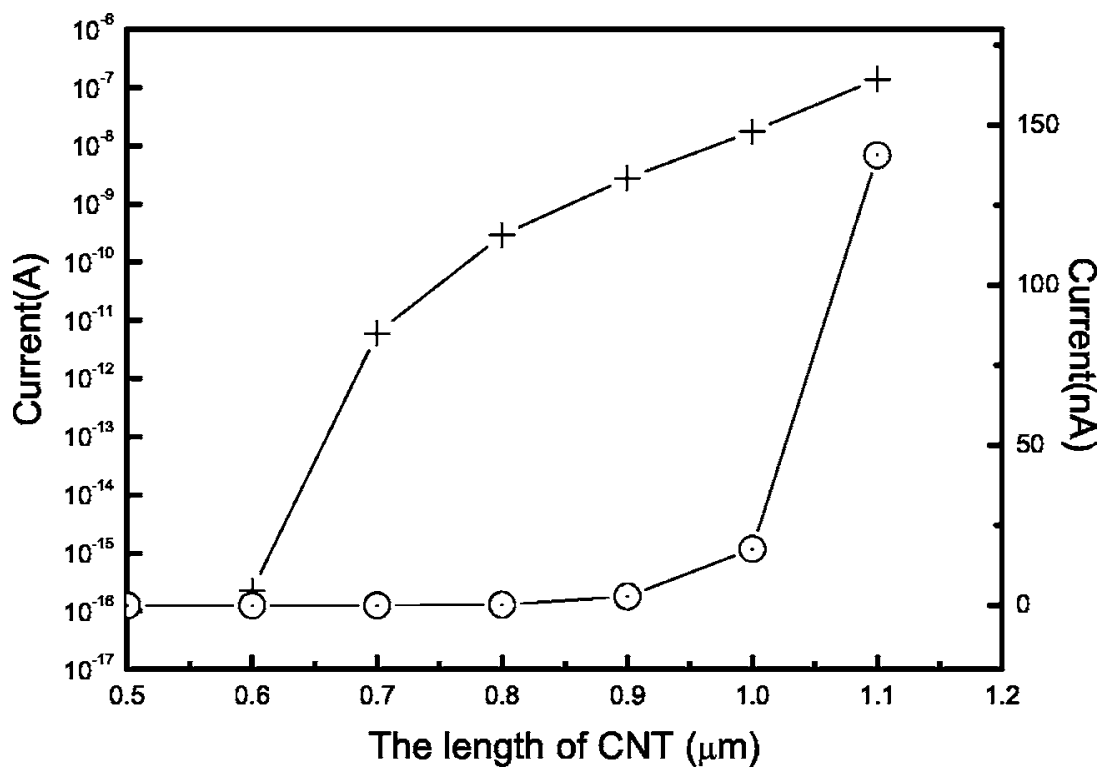

FIG. 7. The current versus length of capped end $(5,5)$ CNTs. The crosses use the left axis that is in logarithmic scale. The circles use the right axis. 


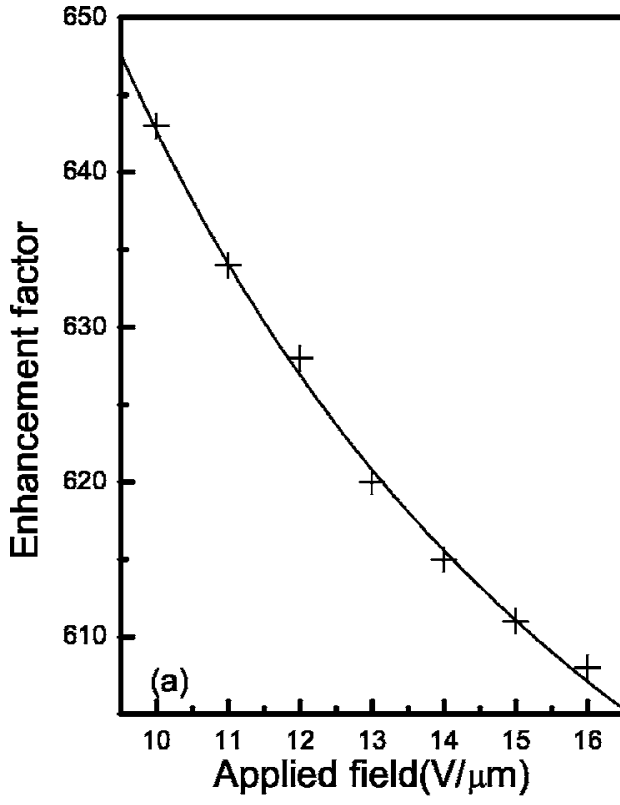

of the apex derived from the electron density distribution. Our result denoted by $\gamma_{q}$ is given in Fig. 8 [(a) for various fields and (b) for various lengths]. It can be fitted by a formula as follows:

$$
\gamma_{q}=\frac{F_{0}}{F_{\text {appl }}}+C \frac{L}{r},
$$

where $F_{0}$ is the field in the apex of the capped SWCNT in the absence of applied field, $F_{\text {appl }}$ is the applied fields, $C$ is a constant, $L$ is the length, and $r$ is the radius of the SWCNT. Our simulations give $F_{0}=950 \mathrm{~V} / \mu \mathrm{m}$ and $C=0.2$. $C$ is smaller than unity reflects the fact that the field penetration reduces the effective shielding length.

\section{CONCLUSIONS}

The emission current versus the applied fields and versus the CNT lengths of experimental scale are simulated, respectively, by a multiscale quantum mechanical method. The FN plot enables us to define a phenomenological field enhancement factor that is consistent with the existing experimental results. However, the consistence should not be understood as evidence of the validity of FN theory for field emission from CNTs. We have pointed out that the phenomenological field enhancement factor is in principle different from the real apex field enhancement factor. Our simulation shows that the real apex field enhancement factor depends on both the applied field and the length. Its magnitude is obviously different from either the phenomenological one and that calculated by a classical model. This indicates that a new mechanism plays a role in the field emission from CNTs.
FIG. 8. (a) The enhancement factor versus the applied fields for the $1.0 \mu \mathrm{m}$ CNT. (b) The enhancement factor versus CNT lengths in $14.0 \mathrm{~V} / \mu \mathrm{m}$.
The charge distribution and the electrostatic potential are determined consistently. The stereo picture of charge distribution indicates clearly the positions most likely to be the outlet for the emitting electrons. It also shows that the electric dipoles at the apex due to the curvature of the cap have influence on the apex-vacuum barrier.

An important observation is that the apex barrier height decreases with respect to the Fermi level under the applied fields. It is also found that the barrier height is lower for a longer SWCNT. These phenomena can be understood as the consequences of the charge accumulation in the tip region. The steplike lowering of the potential barrier should be attributed to the DOS in the tip region. The barrier lowing together with the field enhancement effect turn out to be two main reasons for the efficient field emission of the long SWCNTs.

\section{ACKNOWLEDGMENTS}

The authors gratefully acknowledge financial support of the projects from the National Natural Science Foundation of China (the Distinguished Creative Group Project; Grant No. 90103028, 90306016), from the Education Ministry of China, from the Higher Education Bureau, and from the Science and Technology Commission of Guangdong Province. Zheng and Chen gratefully acknowledge support from the Hong Kong Research Grant Council (Grant No. HKU 7012/ 04P) and the Committee for Research and Conference Grants (CRCG) of the University of Hong Kong. The authors thank Paul Erdoes and Chris Edgcombe for improving the manuscript. 
*Corresponding auther: stsxns@ zsu.edu.cn

†Corresponding author: ghc@everest.hku.hk

${ }^{1}$ W. A. de Heer, A. Châtelain, and D. Ugarte, Science 270, 1179 (1995).

${ }^{2}$ J.-M. Bonard, K. A. Dean, B. F. Coll, and C. Klinke, Phys. Rev. Lett. 89, 197602 (2002).

${ }^{3}$ R. H. Baughman, A. A. Zakhidov, and W. A. de Heer, Science 297, 787 (2002).

${ }^{4}$ A. Modinos, Field, Thermionic, and Secondary Electron Emission Spectroscopy (Plenum Publishing, New York, 1984).

${ }^{5}$ P. G. Collins and A. Zettl, Phys. Rev. B 55, 9391 (1997).

${ }^{6}$ S. Han and J. Ihm, Phys. Rev. B 61, 9986 (2000).

${ }^{7}$ S. Han, M. H. Lee, and J. Ihm, Phys. Rev. B 65, 085405 (2002).

${ }^{8}$ S. Han and J. Ihm, Phys. Rev. B 66, 241402(R) (2002).

${ }^{9}$ G. Zhou and Y. Kawazoe, Chem. Phys. Lett. 350, 386 (2001).

${ }^{10}$ X. Zheng, G. H. Chen, Z. B. Li, S. Z. Deng, and N. S. Xu, Phys.
Rev. Lett. 92, 106803 (2004).

${ }^{11}$ A. Warshel and M. Levitt, J. Mol. Biol. 103, 227 (1976).

${ }^{12}$ R. Saito, G. Dresselhaus, and M. S. Dresselhaus, Physical Properties of Carbon Nanotubes (Imperial College Press, London, 1998).

${ }^{13}$ John Cumings, A. Zettl, M. R. McCartney, and J. C. H. Spence, Phys. Rev. Lett. 88, 056804 (2002).

${ }^{14}$ R. Tamura and M. Tsukada, Phys. Rev. B 52, 6015 (1995).

${ }^{15}$ D. L. Carroll, P. Redlich, P. M. Ajayan, J. C. Charlier, X. Blase, A. De Vita, and R. Car, Phys. Rev. Lett. 78, 2811 (1997).

${ }^{16}$ W. Yang, Phys. Rev. Lett. 66, 1438 (1991).

${ }^{17}$ G. C. Kokkorakis, A. Modinos, and J. P. Xanthakis, J. Appl. Phys. 91, 4580 (2002).

${ }^{18}$ C. J. Edgcombe and U. Valdre, Solid-State Electron. 45, 857 (2001). 